\title{
Calidad de cocción, aceptabilidad e índice glicémico de pasta larga enriquecida con leguminosas
}

\author{
Quality of cooking, acceptability \\ and glycemic index of enriched \\ pasta with legumes
}

\begin{abstract}
Low glycemic index diets have been shown to improve glucose tolerance in healthy and diabetic people. However, it is necessary to diversify the diet with foods with low glycemic response. In this study, three partially substituted pasta made with semolina and flour legumes (12\% Phaseolus vulgaris, Vigna sinensis $10 \%, 12 \%$ of Cajanus cajan) were formulated and its cooking quality, acceptability, glycemic index and glycemic load were assessed. We found that the incorporation of legumes impacts the cooking quality and sensory characteristics of the product, increasing cooking losses between $78 \%$ and $557 \%$, and the content of soluble proteins between $14 \%$ and $32 \%$. In relation to the sensory characteristics, increases in hardness and stickiness were observed, without altering the overall appreciation (9 on a scale of 0 to 10). An Improvement of on the nutritional quality was observed, increasing the protein content between $19 \%$ and $26 \%$. The glycemic index value found for the three pasta formulations resemble the type of slow and intermediate carbohydrate absorption.
\end{abstract}

Key words: legumes; pasta; glycemic index; f cooking quality; sensory evaluation.
Marisela Granito (1)

Suhey Pérez (2)

Yolmar Valero (1)

(1) Departamento de Tecnología de Procesos Biológicos y Bioquímicos. Universidad Simón Bolívar. Caracas, Venezuela. (2) Departamento de Tecnología de Servicios. Universidad Simón Bolívar. Caracas, Venezuela.

Dirigir la correspondencia a: Profesora

Marisela Granito

Departamento de Tecnología de Procesos Biológicos y Bioquímicos Universidad Simón Bolívar Caracas, Venezuela

E-mail:mgranito@usb.ve

Este trabajo fue recibido el 19 de Marzo de 2014, aceptado con modificaciones el 1 de Agosto de 21014 y aceptado para ser publicado el 15 de Octubre de 2014.

del producto (3)

Dependiendo del tipo de carbohidrato presente en la pasta, su consumo no sólo regula el IG de la dieta ingerida sino el metabolismo lipídico del consumidor (4). Una de las razones por las cuales ocurre es por la presencia de almidón resistente (AR) y fibra soluble (FS). EI AR no solamente beneficia la salud a través de la producción de ácidos grasos de cadena corta, producto de su fermentación colónica, sino que contribuye directamente con la disminución de la glucosa y la insulina plasmática (5). Behall y colaboradores (6), encontraron una disminución en la glucosa e insulina postprandiales después del consumo de una comida que aportaba 11,5 g de AR (6), mientras que Yamada y colaboradores (7) reportaron respuestas glicémicas e insulinémicas disminuidas después del consumo de $6 \mathrm{~g}$ de AR. Asimismo, Noakes y colaboradores (8) encontraron disminuciones en la glicemia e insulina postprandial en individuos hipertriglicéridémicos después del consumo de 5,8 g de AR. Por su parte la fibra dietética, particularmente la fibra soluble, disminuye el IG al enlentecer la velocidad de absorción intestinal de la glucosa, a través da la formación de estos elementos estructurales a través de la adición de una leguminosa puede inducir a un cambio en el índice glicémico 
un gel viscoso en la pared absortiva del intestino (9).

Una ración de $250 \mathrm{~g}$ de pasta industrial de sémola cocida aporta aproximadamente $57 \mathrm{~g}$ de carbohidratos totales, $11 \mathrm{~g}$ de proteínas y 2,2 g de AR, tipo AR3 o almidón retrogradado, el cual, junto a la fibra dietética presente genera un índice glicémico de $64 \%$, convirtiéndola así en un excelente alimento para personas diabéticas o con resistencia a la insulina (5). Sin embargo, de acuerdo a Ulmius y colaboradores (10) el IG de un alimento no sólo es producto de la presencia de fibra dietética, sino del tamaño de partícula de la fuente de fibra y de la interacción de esta con la proteína y tipo de aminoácidos presentes en la matriz del alimento que aporta la fibra. Aminoácidos ramificados como la leucina, isoleucina y valina, incrementan el IG.

Al formular una pasta, la sémola puede ser sustituida parcialmente por otros ingredientes de alto contenido nutricional como las leguminosas, incrementándose los efectos nutritivos y funcionales de la misma. En general, las leguminosas contienen $18 \%$ a $25 \%$ de proteína y $50 \%$ a $60 \%$ de carbohidratos, entre los cuales predominan el almidón, la fibra dietética y el almidón resistente (11).

Las leguminosas contienen tanto AR1 (almidón físicamente inaccesible), AR2 (almidón no digerible) y AR3 (almidón retrogradado). Con el procesamiento térmico, disminuye el AR1 y el AR2 y se incrementa el AR3. El alto contenido de AR de las leguminosas explica, al menos parcialmente, porque la tasa de digestión y por tanto la liberación de la glucosa en la sangre es más lenta, resultando en una respuesta glicémica e insulinémica reducida en comparación con los cereales o tubérculos (12-14).

De acuerdo a Cassab y colaboradores (15) Phaseolus vulgaris cocida contiene $4,75 \%$ de AR y un IG de 38\%, mientras que Osorio-Díaz y colaboradores (16) reportaron incrementos de $24 \%$ en el AR y descensos de $10 \%$ en el IG para espaguetis de sémola sustituidos por $20 \%$ de harina de garbanzo. A pesar de que el procesamiento térmico disminuye el AR1 y el AR2, se han reportado para leguminosas cocidas y enlatadas contenidos entre 9,5 y $11,1 \%$ de almidón resistente (17), probablemente gracias al incremento del almidón retrogradado (AR3) el cual se forma producto de los diferentes ciclos de calentamiento y enfriamiento. Sin embargo, de acuerdo a Landa y colaboradores (18) el almacenamiento de Phaseolus vulgaris cocida a $4^{\circ} \mathrm{C}$ por $96 \mathrm{~h}$ incrementa el contenido de AR3.

Sin embargo, para comprobar que la presencia de $A R$ reduce el IG, en adición a la determinación de la respuesta glucémica obtenida por los productos control y una cantidad de producto de prueba que contienen la misma cantidad de carbohidratos totales, se hace un ensayo adicional con una cantidad de producto prueba que contenga una cantidad igual de carbohidratos disponibles como el control (19). Jenkins y colaboradores (20) hicieron esto y encontraron que el efecto sobre la glicemia reducida fue debido a la reducción en la cantidad de almidón absorbido sin ningún efecto sobre el IG.

Según la International Pasta Organization (21), en 2011 Venezuela era el segundo consumidor de pasta a nivel mundial, con un consumo aproximado de 12,3 Kg per cápita al año. Por otra parte, las leguminosas son alimentos altamente disponibles, así es posible inferir que las pastas cuya sémola sea parcialmente sustituida por harinas de leguminosas podrían convertirse en una alternativa de consumo de un alimento con índice glicémico inferior al de las pastas de sémola, dirigido a personas con resistencia a la insulina y diabéticos.

El objetivo de este trabajo fue formular pastas de sémola parcialmente sustituidas con harina de leguminosas y evaluar la calidad de cocción, aceptabilidad, índice glicémico y carga glicémica de las mismas.

\section{MATERIALES Y MÉTODOS}

Materias primas: La sémola de trigo durum (STD) y las leguminosas Phaseolus vulgaris, Vigna sinensis y Cajanus cajan, obtenidas en el comercio local.

Leguminosas: las leguminosas fueron cocidas a presión atmosférica, cada una por separado, a temperaturas de ebullición durante aproximadamente 120 minutos. Posteriormente, se drenó el agua de cocción y se homogenizaron en una licuadora industrial (OSTER, USA) hasta un tamaño de partícula entre 150 y $340 \mu \mathrm{m}$. El homogenizado fue almacenado a $4^{\circ} \mathrm{C}$ por $96 \mathrm{~h}$ y 15 minutos antes de su utilización fue colocado a temperatura ambiente y posteriormente añadido a la sémola para la elaboración de la pasta.

Formulación de las pastas: se formularon 3 variedades de pasta larga, tipo espagueti, sustituyendo la sémola de trigo por $12 \%$ de Phaseolus vulgaris, 10\% de Vigna sinensis, 12\% de Cajanus cajan, tomando en consideración investigaciones reportadas en la literatura (22). Adicionalmente se preparó una pasta control con $100 \%$ sémola de trigo.

A la mezcla de sémola y cada una de las leguminosas, por separado, se les adicionó agua (35-40\%) para el amasado, se dejó en reposo y posteriormente la masa fue procesada en una máquina para elaborar pastas, tipo espagueti. Las pastas frescas fueron secadas a temperatura ambiente (1h) y luego por $24 \mathrm{~h}$ a $30^{\circ} \mathrm{C}$ en estufa de convección, hasta alcanzar una humedad del $12 \%$. Las pastas fueron conservadas en bolsas herméticas bajo refrigeración hasta su análisis.

Evaluación sensorial: Una vez cocidas, la pasta se escurrió y se sirvió aún caliente y sin ningún aderezo. Se utilizó un panel de 50 miembros semientrenados quienes evaluaron la dureza, pegajosidad y volumen utilizando una escala no estructurada de $10 \mathrm{~cm}$, con anclajes extremos de "poco" y "extremadamente". La apreciación global de la pasta fue evaluada usando una escala hedónica no estructurada de 10 $\mathrm{cm}$, con anclajes extremos de "me disgusta mucho" y "me agrada mucho". A cada panelista se le presentó cada una de las pastas por separado.

Pruebas de cocción: La calidad de cocción de la pasta se determinó de acuerdo a Abecassis y colaboradores (23) y Matsuo y colaboradores (24) utilizando las siguientes condiciones:

Tiempo de cocción: se dispersaron $10 \mathrm{~g}$ de pasta en 100 $\mathrm{ml}$ de agua en ebullición. Cada minuto, se tomaba un trozo de pasta entre dos vidrios de reloj y se comprimía. El tiempo óptimo de cocción (min) se estableció como aquel al cual desaparecía el color blanco después de la compresión.

Absorción del agua de cocción: la pasta cocida fue drenada y se pesó antes y después de la cocción.

Pérdidas por cocción: los sólidos extraídos del agua de cocción fueron calculados concentrando el agua de cocción hasta sequedad en un horno a $100^{\circ} \mathrm{C}$.

Pérdida de proteína en el agua de cocción: fue determinado cuantificando la proteína del agua de cocción a través del método de Biuret, descrito por Robinson y Hodgen (25)

Composición proximal: La composición proximal de las pastas elaboradas: humedad, proteínas, grasa y cenizas fueron cuantificadas a través de los métodos AOAC 925.09, 960.52, 920.39, 923.03, respectivamente (26).

El contenido de fibra dietética se determinó siguiendo el procedimiento descrito por Goñi y colaboradores (27). El almidón total se determinó por hidrólisis enzimática, de acuerdo a la metodología descrita por Goñi y colaboradores (28) y 
el contenido de almidón resistente se determinó de acuerdo a la metodología descrita por Goñi y colaboradores (29). La cantidad de almidón disponible fue calculado por diferencia.

Determinación del índice glicémico: Se siguió el procedimiento descrito en la literatura $(30,31)$. La población escogida para esta prueba estuvo conformada por 9 adultos sanos (4 hombres-5 mujeres), con edades comprendidas entre 25 y 40 años de edad, que no padecieran de diabetes mellitus o alguna enfermedad crónica (tabla 1). Las variables antropométricas utilizadas como criterios de inclusión fueron peso, talla e índice de masa corporal (IMC), calculado a partir de los dos primeros. Se realizó una encuesta para asegurar que los individuos no estuviesen ingiriendo ningún medicamento o suplemento dietético o estuviesen embarazadas.

Los sujetos leyeron y firmaron un consentimiento con información escrita acerca del protocolo, de acuerdo a lo establecido por la Comisión de Ética de la Universidad Simón Bolívar, siguiendo las consideraciones bioéticas estipuladas en la Declaración de Helsinki y ratificadas por el Fondo Nacional de Ciencia, Tecnología e Innovación. Los sujetos fueron distribuidos al azar y se les practicaron las curvas de tolerancia glucosada con intervalo de una semana entre cada prueba. La primera evaluación se realizó con el alimento de referencia (pan blanco, cantidad suficiente para ingerir $50 \mathrm{~g}$ de carbohidratos) y las siguientes semanas sucesivas las tres pastas formuladas y la pasta control (cantidad suficiente para ingerir $50 \mathrm{~g}$ de carbohidratos). Cada una de estas evaluaciones se realizó por duplicado. El día de la prueba, las muestras fueron consumidas en un tiempo máximo entre 10-12 min después de la toma basal. Fue autorizado el consumo hasta un máximo de 250 $\mathrm{ml}$ de agua durante la ingesta. Una muestra de sangre capilar fue tomada usando una lanceta (Accu-chek Softclix, Roche
Diagnostics, España) en el tiempo 0 (basal) y a los 15, 30, 45, 60,90 y 120 min, después de la ingesta de cada uno de los alimentos analizados. Las muestras de sangre fueron captadas en tiras reactivas y analizadas con un equipo analizador de glucosa (Accutrend-Sensor, Roche Diagnostics, España). Con las concentraciones de glucosa, expresadas como promedio \pm desviación estándar, se determinó el área bajo la curva ( $A B C)$, calculada con los valores por encima de la línea de base en el período de $2 \mathrm{~h}$ después de ingerido el alimento.

Carga glicémica (CG): La CG representa una medida derivada del valor del IG del alimento en estudio y se calcula de acuerdo a la siguiente fórmula (32):

Donde:

$$
C G=\frac{I G \times C H O \text { por porción de alimento }}{100}
$$

IG: índice glicémico del alimento a evaluar.

CHO: carbohidratos presente en el alimento por porción.

Los valores resultantes han sido categorizados en CG alta $>20$, CG media 11-19 y CG baja $<10$.

Análisis estadístico: Los datos se presentan como el promedio de tres determinaciones \pm la desviación estándar. Los datos fueron analizados estadísticamente usando análisis de varianza, empleando el programa Statgraphics 5.0.

\section{RESULTADOS}

\section{Evaluación sensorial}

La tabla 2 muestra los resultados de la evaluación sensorial de las pastas formuladas. Se observa que la sustitución con leguminosas incrementó significativamente $(P<0,05)$ la dureza y pegajosidad del producto.

\section{TABLA 1}

Características e indicadores bioquímicos de ingreso de los participantes en el estudio de índice glicémico.

$\mathrm{N}^{\circ}$ de sujetos

Sexo

Edad (años)

Peso $(\mathrm{Kg})$

Talla $(\mathrm{m})$

Índice de masa corporal $(\mathrm{Kg} / \mathrm{m} 2)$

Glucosa inicial (mg/dl)
9

5 mujeres / 4 varones

$$
\begin{gathered}
37,02 \pm 3,67 \\
66,62 \pm 3,66 \\
1,67 \pm 0,07 \\
22,79 \pm 3,11 \\
93,2 \pm 3,03
\end{gathered}
$$

TABLA 2

Evaluación sensorial de las pastas desarrolladas.

\begin{tabular}{lcccc}
\hline & PC & PVS & PPV & PCC \\
Dureza & $4,8 \pm 1,2^{\mathrm{a}}$ & $6,9 \pm 0,8^{\mathrm{b}}$ & $6,7 \pm 0,5^{\mathrm{b}}$ & $5,1 \pm 1,0^{\mathrm{b}}$ \\
Pegajosidad & $3,1 \pm 0,7$ & $4,3 \pm 0,9$ & $6,1 \pm 0,5$ & $7,7 \pm 0,4$ \\
Apreciación Global & $8,7 \pm 0,6^{\mathrm{a}}$ & $8,4 \pm 0,3^{\mathrm{a}}$ & $8,5 \pm 0,4^{\mathrm{a}}$ & $9,3 \pm 0,2^{\mathrm{b}}$ \\
\hline
\end{tabular}

Letras diferentes en una misma fila indican diferencias estadísticamente significativas. PC pasta formulada con 100\% semola de trigo,

PVS pasta formulada con $10 \%$ de Vigna sinensis. PPV pasta formulada con $12 \%$ de Phaseolus vulgaris, PCC pasta formulada con $12 \%$ de Cajanus cajan. 
La mayor apreciación global fue de la pasta sustituida con Cajanus cajan. Sin embargo, tal como se observa en todos los casos la apreciación global obtuvo puntuación por encima de 7, en una escala no estructurada de $10 \mathrm{~cm}$.

\section{Pruebas de cocción}

En la tabla 3 se observa la calidad de cocción de las pastas formuladas, la sustitución con leguminosas incrementó los tiempos de cocción entre un 13,3\% y 34,8\%. Resultados similares fueron reportados por Granito y Ascanio (22).

La absorción de agua no presentó diferencias significativas en las muestras sustituidas con Phaseolus vulgaris y Cajanus cajan, respecto a la pasta sin sustitución. Sin embargo, la pasta sustituida con Vigna sinensis mostró un incremento de 7,4\% respecto a la pasta formulada únicamente con sémola.

Respecto a la pasta control, las pérdidas por cocción en las pastas sustituidas con leguminosas, se incrementaron entre $78 \%$ y $557 \%$.

\section{Composición proximal}

En la tabla 4 se presentan los resultados de la evaluación química de las pastas preparadas. El contenido de humedad de las tres muestras preparadas con leguminosa fue mayor a la de la pasta sin sustitución, sin embargo cumplen con las especificaciones de humedad establecidas en la Norma Venezolana COVENIN 283:1994 para pastas alimenticias de 13,5 (\% máximo) y 12,8 (\%mínimo), respectivamente (33), los niveles de humedad obtenidos demuestran un adecuado secado.

La sustitución de la sémola con leguminosas incrementó el contenido de proteínas entre $19 \%$ y $26 \%$. En el contenido de cenizas también se observó un incremento que fue entre $22 \%$ y $111 \%$, respecto a la PC. Por otra parte, el contenido de grasas disminuyo en $97 \%$.

El contenido de fibra soluble en las pastas sustituidas con leguminosa presentó un incremento entre 38\% y 98\%, respecto a la PC. La fibra insoluble se incrementó entre un $69 \%$ y $124 \%$. Finamente el contenido de la fibra total, se incremento hasta $102 \%$ respecto a la pasta sin sustitución.

El contenido de almidón total, resistente y disponible también se incrementó significativamente $(P<0,05)$ en las pastas sustituidas con leguminosa respecto a la pasta sin sustitución.

\section{Determinación del índice glicémico}

La figura 1 muestra el incremento de glucosa en sangre en sujetos sanos posterior a la ingesta de pan blanco (alimento

\section{TABLA 3}

Calidad de cocción de las pastas formuladas.

\begin{tabular}{|c|c|c|c|c|}
\hline & PC & PVS & PPV & PCC \\
\hline Tiempo de cocción (min) & $15,00 \pm 1,00^{\mathrm{a}}$ & $23 \pm 1,00 c$ & $17 \pm 2,00^{a}$ & $19 \pm 2,00^{b}$ \\
\hline Absorción de agua (\%) & $172,20 \pm 3,55^{\mathrm{a}}$ & $185,04 \pm 0,64^{b}$ & $171,07 \pm 0,31^{\mathrm{a}}$ & $169,54 \pm 0,71^{\mathrm{a}}$ \\
\hline Pérdidas por cocción (\%) & $3,00 \pm 0,15^{a}$ & $5,35 \pm 0,15^{b}$ & $15,13 \pm 0,12^{c}$ & $19,7 \pm 0,08^{d}$ \\
\hline Proteínas solubles (mg/g pasta cruda) & $15,0 \pm 0,01^{\mathrm{a}}$ & $19,85 \pm 0,21^{c}$ & $18,26 \pm 0,41^{c}$ & $17,16 \pm 0,52^{b}$ \\
\hline
\end{tabular}

\section{TABLA 4}

Composición proximal de las pastas formuladas.

\begin{tabular}{lcccc}
\hline Análisis (g/100g) & PC & PVS & PPV & PCC \\
Humedad & $7,2 \pm 0,56^{\mathrm{a}}$ & $13,20 \pm 0,21^{\mathrm{c}}$ & $12,10 \pm 0,31^{\mathrm{b}}$ & $12,40 \pm 0,24^{\mathrm{b}}$ \\
Proteínas & $16,29 \pm 0,37^{\mathrm{a}}$ & $20,49 \pm 0,15^{\mathrm{c}}$ & $19,36 \pm 0,37^{\mathrm{b}}$ & $18,02 \pm 0,41^{\mathrm{b}}$ \\
Grasas & $1,78 \pm 0,10^{\mathrm{c}}$ & $0,06 \pm 0,01^{\mathrm{a}}$ & $0,05 \pm 0,01^{\mathrm{a}, \mathrm{b}}$ & $0,04 \pm 0,01^{\mathrm{a}}$ \\
Cenizas & $0,55 \pm 0,02^{\mathrm{a}}$ & $1,16 \pm 0,08 \mathrm{~d}$ & $0,67 \pm 0,02^{\mathrm{a}}$ & $0,95 \pm 0,06^{\mathrm{c}}$ \\
Fibra soluble & $2,77 \pm 0,53^{\mathrm{a}}$ & $3,83 \pm 0,11^{\mathrm{b}}$ & $5,49 \pm 0,1^{\mathrm{c}}$ & $4,33 \pm 0,11^{\mathrm{d}}$ \\
Fibra insoluble & $5,66 \pm 0,63^{\mathrm{a}}$ & $11,69 \pm 0,18^{\mathrm{c}}$ & $9,55 \pm 0,17^{\mathrm{b}}$ & $12,7 \pm 0,2^{\mathrm{d}}$ \\
Fibra total & $8,43 \pm 0,58^{\mathrm{a}}$ & $15,51 \pm 0,1^{\mathrm{c}}$ & $15,04 \pm 0,1^{\mathrm{b}}$ & $17,03 \pm 0,22^{\mathrm{d}}$ \\
Almidón total & $50,14 \pm 0,5^{\mathrm{a}}$ & $69,40 \pm 0,13^{\mathrm{b}}$ & $71,7 \pm 0,43^{\mathrm{c}}$ & $79,68 \pm 0,06^{\mathrm{d}}$ \\
Almidón resistente & $2,02 \pm 0,15^{\mathrm{a}}$ & $9,95 \pm 0,2^{\mathrm{b}}$ & $10,43 \pm 0,21^{\mathrm{c}}$ & $11,07 \pm 0,23^{\mathrm{d}}$ \\
Almidón disponible & $48,12 \pm 0,34^{\mathrm{a}}$ & $59,45 \pm 1,16^{\mathrm{b}}$ & $61,23 \pm 1,24^{\mathrm{b}}$ & $68,62 \pm 0,29^{\mathrm{c}}$ \\
\hline
\end{tabular}

Letras diferentes en una misma fila indican diferencias estadísticamente significativas $\mathrm{P}<0,05$. $\mathrm{PC}$ pasta formulada con $100 \%$ semola de trigo,

PVS pasta formulada con $10 \%$ de Vigna sinensis. PPV pasta formulada con 12\% de Phaseolus vulgaris, PCC pasta formulada con $12 \%$ de Cajanus cajan. 
de referencia), la pasta cocida sin sustitución y las tres pastas sustituidas con leguminosas, para la estimación del índice glicémico.

El mayor incremento de las curvas de glucosa se observó con el alimento estándar, a los 15 y 30 minutos, comenzando el descenso a partir de los 45 minutos y volviendo a su nivel basal (glicemia en ayunas) al finalizar el tiempo de prueba. El incremento de la curva correspondiente a la pasta cocida sin sustitución presentó un comportamiento similar que el alimento de referencia, pero con ascenso a los 15, 30 y 45 minutos posteriores a la ingesta, con incrementos menores a los reportados para el pan blanco, y descenso a partir de los 60 minutos y llegando nuevamente a su valor basal de inicio de la prueba.

Para la pasta cocida con $10 \%$ de Vigna sinensis el incremento observado presenta un pico máximo de glucosa los 30 minutos, luego desciende gradual y significativamente, con respecto al alimento estándar, a partir de los $45 \mathrm{~min}$, hasta su valor basal de inicio de prueba.

La pasta cocida con $12 \%$ de Phaseolus vulgaris y la pasta cocida con $12 \%$ de Cajanus cajan presentaron el mismo comportamiento entre ambas, con un incremento de la glucosa hasta el minuto 45 y descenso gradual hasta valores cercanos al valor basal para la pasta cocida con $12 \%$ de Cajanus cajan, y ligeramente menor al valor a tiempo 0, a los 120 minutos de la ingesta, para la pasta cocida con $12 \%$ de Phaseolus vulgaris.

Las concentraciones máximas de glucosa fueron de 129 $\pm 2,56 \mathrm{mg} / \mathrm{dl}, 124 \pm 3,89 \mathrm{mg} / \mathrm{dl}$ y $120 \pm 2,45 \mathrm{mg} / \mathrm{dl}$ para las pastas cocidas sin sustitución, con $12 \%$ de Phaseolus vulgaris y con $12 \%$ de Cajanus cajan, a los 45 min, respectivamente y de $126 \pm 3,01 \mathrm{mg} / \mathrm{dl}$ para la pasta con $10 \%$ de Vigna sinensis, a los $30 \mathrm{~min}$.

Con los datos obtenidos del ABC del alimento estándar y las pastas evaluadas se obtuvo el valor de índice glicémico y carga glicémica, como se observa en la tabla 5. El índice glicémico obtenido para la pasta cocida sin sustitución fue de 76, considerado como un alimento de índice glicémico alto. Las pastas cocidas con $10 \%$ de Vigna sinensis, con $12 \%$ de Phaseolus vulgaris y con $12 \%$ de Cajanus cajan presentaron una disminución del IG de 17\%, 20\% y 22\% respectivamente, con relación a la pasta cocida sin sustitución. En relación a la carga glicémica, todas las pastas formuladas y la pasta sin sustitución presentaron una carga glicémica alta, sin embargo, esta carga fue mayor (17,5\% aproximadamente) para la pasta sin sustitución, que para las pastas sustituidas con las leguminosas.

\section{FIGURA 1}

Incremento del valor de glucosa (promedio de dos repeticiones) en sangre en sujetos sanos posterior a la ingesta de pan blanco.

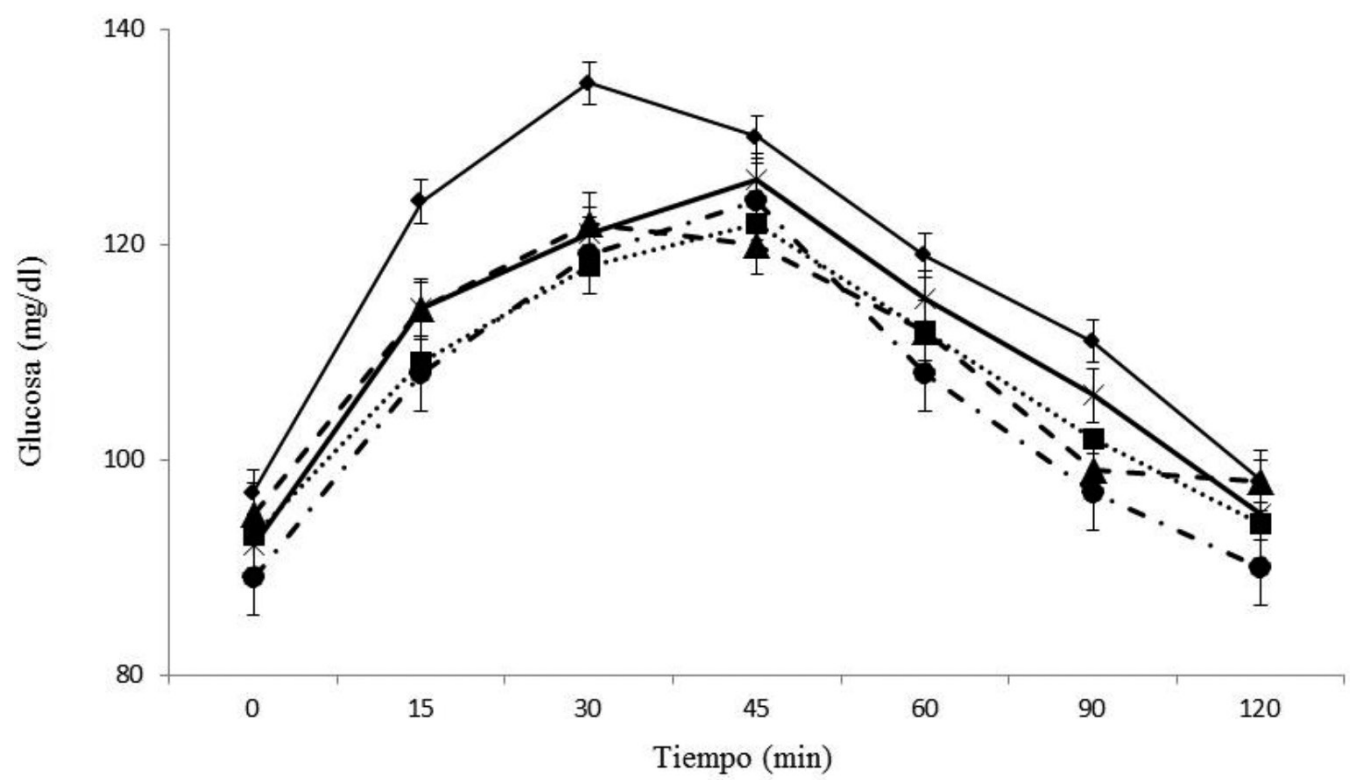

$(-\downarrow-)$ pasta cocida de semola

$(-x-)$ pasta cocida con $10 \%$ de Vigna sinensis

(-A-) pasta cocida con $12 \%$ de Phaseolus vulgaris

(.. ...) y pasta cocida con $12 \%$ de Cajanus cajan

(-•-) para la estimación del índice glicémico. 


\section{DISCUSIÓN}

Evaluación sensorial

La modificación de la dureza y pegajosidad de pastas también fueron reportadas Zhao y colaboradores (34) y Petitot y colaboradores (3), quienes encontraron que al aumentar el nivel de sustitución de sémola por harina o concentrado proteico de leguminosa se incrementa la dureza, debido al aumento del contenido de proteína en el producto.

Martín y colaboradores (35) indican que al incrementar el contenido de fibra se incrementa la absorción de agua y con ello la dureza de la pasta. Al respecto es importante mencionar que las proteínas de la sémola, predominantemente glidianas y gluteninas, interactúan a través de puentes disulfuro y de hidrógeno formando una red tridimensional, responsable de la textura de la pasta (36). En contraste, las proteínas de las leguminosas son predominantemente globulinas solubles en sal y albuminas solubles en agua (37). La incorporación de proteínas que no son fuente de gluten, debilitan la red y generan desintegración de la pasta durante la cocción, incrementando la cohesividad y pegajosidad del producto (38).

\section{Pruebas de cocción}

El incremento en la absorción de agua en la pasta formulada con Vigna sinenses puede atribuirse a la disrupción física de la matriz de gluten por la fibra de la leguminosa, lo cual podría facilitar la penetración del agua al centro de la pasta (39). La modificación tanto de los tiempos de cocción, como de la absorción de agua, depende adicionalmente del diámetro del espagueti y la temperatura de secado.

La pérdida de sólidos disueltos no es deseable, porque es producida por la solubilización de compuestos como almidones, proteínas y minerales, los cuales pasan al agua de cocción. Esta pérdida de sólidos ocasiona que las pastas pierdan su forma si se dejan más tiempo sumergidas en agua caliente. Las pérdidas por cocción pueden deberse a lixiviación de amilosa o solubilización de proteínas solubles y sales (3). Aun cuando la sustitución con leguminosas incrementa significativamente $(p<0,05)$ el contenido de proteínas en la pasta, también se incrementa significativamente $(p<0,05)$ las proteínas solubles, lo anterior debido a la naturales hidrofílica de las proteínas incorporadas.

\section{Composición proximal}

La sustitución con harina de leguminosa podría mejorar la calidad de proteína en el producto, debido a la complementación aminoacídica entre cereales y leguminosas (31). Resultados similares han sido reportados por Zhao y colabo- radores (34) y Petitot y colaboradores (3).

El contenido de grasa de las pastas sustituidas fue menor que el indicado en la Tabla de Composición de Alimentos (40) $(1,9 \%)$, lo que podría favorecer la estabilidad durante el almacenamiento del producto.

El contenido de cenizas de la pasta sustituida con 10\% de Vigna sinensis fue $16 \%$ mayor a lo establecido por la norma, con lo cual se puede inferir que la sustitución incrementa el aporte de minerales de la pasta.

La sustitución con harinas de leguminosa incrementó significativamente $(p<0.05)$ el contenido de fibra y almidones, lo cual implica aumento en el contenido carbohidratos de absorción lenta, los cuales tienen efecto sobre la respuesta glicémica del producto.

Determinación del índice glicémico

Con base en el concepto que los carbohidratos simples (mono y disacáridos) inducían un incremento de la glicemia más rápido y mayor que los complejos (polisacáridos) y que los distintos alimentos, independiente de su contenido total, presentan una proporción diferente de carbohidratos, entre simples y complejos (32), nace el concepto de índice glicémico.

El valor de índice glicémico encontrado para las tres pastas extendidas con leguminosas, desarrolladas, corresponden al tipo de carbohidratos de absorción lenta e intermedia. La respuesta glicémica a una comida, que a su vez afecta a la respuesta de la insulina, va a depender de la tasa de vaciado gástrico, así como en la velocidad de digestión y la absorción carbohidratos en el intestino delgado. Los carbohidratos de absorción lenta han sido considerados ventajosos para los individuos sanos, para evitar una respuesta excesiva de insulina e hipoglucemia entre las comidas. En el caso de las personas diabéticas o con síndrome de resistencia a la insulina, estos deben seleccionar, para su consumo, alimentos con carbohidratos de absorción lenta, que ayuda a estos pacientes para mejorar su control de la glucosa sanguínea (41).

Se ha demostrado que la pasta, como alimento, puede tener propiedades beneficiosas en el mantenimiento de la salud. Goñi y Valentin-Gamazo (31) evaluaron dos tipos de pasta de producción industrial. Una de las pastas se elaboró con $100 \%$ de harina de trigo durum, la otra a partir de una mezcla (75/25) de harina de trigo durum y harina de garbanzo. El índice glicémico para los dos tipos de pasta se encontró dentro del rango normal de carbohidratos de absorción lenta, pero el índice fue significativamente menor para la pasta extendida con harina de garbanzo (IG trigo: $73 \pm 5$; IG trigogarbanzo: $58 \pm 6$ ). Se demostró que las pastas alimenticias con

\section{TABLA 5}

Índice glicémico y carga glicémica de las pastas formuladas.

\begin{tabular}{lcccc}
\hline & & Tamaño de la porción habitual & CHO disponibles \\
$(\mathrm{g})$ & & \multicolumn{2}{c}{ Carga glicémica } \\
PC & IG & 75 & 55,63 & $57,0 \pm 1,3^{\mathrm{b}}$ \\
PVS & $76 \pm 2,6^{\mathrm{b}}$ & 75 & 47,31 & $48,7 \pm 3,2^{\mathrm{a}}$ \\
PPV & $65 \pm 3,1^{\mathrm{a}}$ & 75 & 50,86 & $47,3 \pm 2,3^{\mathrm{a}}$ \\
PCC & $63 \pm 2,9^{\mathrm{a}}$ & 75 & 51,44 & $46,5 \pm 1,8^{\mathrm{a}}$ \\
\hline
\end{tabular}

Letras diferentes en una misma columna indican diferencias estadísticamente significativas $\mathrm{P}<0,05$. $\mathrm{PC}$ pasta formulada con $100 \%$ semola de trigo,

PVS pasta formulada con $10 \%$ de Vigna sinensis. PPV pasta formulada con $12 \%$ de Phaseolus vulgaris, PCC pasta formulada con $12 \%$ de Cajanus cajan. 
harina de leguminosas presentan una respuesta glicémica baja, y podría ayudar a ampliar la gama de alimentos de IG bajo a disposición del consumidor.

Sin embargo, desde el punto de vista tecnológico, la pasta presenta diferencias de acuerdo a sus características estructurales. Primeramente, la forma del producto puede variar considerablemente cómo afecta la respuesta glicémica. Las pastas tipo macarrón producen una respuesta glicémica mas alta que las pastas tipo espagueti, probablemente debido a una mayor superficie y densidad de producto (42). Adicionalmente, las diferentes temperaturas de secado a las que es sometido el producto tienen influencia directa en la respuesta glicémica.

\section{CONCLUSIONES}

La sustitución con harina de leguminosas a nivel sensorial incrementa la dureza y pegajosidad de las pastas. Por otra parte, se incrementa el tiempo y pérdidas de cocción, debido principalmente a la incorporación de proteínas no formadoras de gluten y fibras. Por otra parte la calidad nutricional del producto mejora, toda vez que se incrementa el contenido de proteína. El valor de índice glicémico encontrado para las tres pastas extendidas con leguminosas, desarrolladas, corresponden al tipo de carbohidratos de absorción lenta e intermedia.

\section{RESUMEN}

Dietas con índices glicémicos bajos han demostrado mejorar la tolerancia a la glucosa en personas sanas y diabéticas. Sin embargo, es necesario diversificar los alimentos con bajas respuestas glicémicas. En este estudio se formularon tres pastas de sémola parcialmente sustituidas con harina de leguminosas (12\% Phaseolus vulgaris, 10\% de Vigna sinensis, 12\% de Cajanus cajan) y evaluaron la calidad de cocción, aceptabilidad, índice glicémico y carga glicémica de las mismas. Se encontró que la incorporación de leguminosas tiene impacto en la calidad de cocción y características sensoriales del producto, incrementando significativamente las pérdidas por cocción, entre un $78 \%$ y $557 \%$, y el contenido de proteínas solubles, entre 14\% y 32\%. En relación a las características sensoriales, aumenta la dureza y pegajosidad del producto, sin modificar la apreciación general del mismo, 9 en una escala de 0 a 10 . La calidad nutricional del producto mejora, toda vez que se incrementa entre $19 \%$ y $26 \%$ el contenido de la proteína. El valor de índice glicémico encontrado para las tres formulaciones de pastas, corresponden al tipo de carbohidratos de absorción lenta e intermedia.

Palabras clave: leguminosas; pasta; índice glicémico; calidad de cocción; evaluación sensorial.

\section{BIBLIOGRAFÍA}

1. Granfeldt, Y. 1994. Foods factors affecting metabolic responses to cereal products. University of Lund. Sweden. (Ph.D. Thesis).

2. Jenkins $D$, Wolever, $T$, Taylor, $R m$ Barker $H$, Fielden $H$, Baldwin J, Bowling A, Newman H, Jenkins A, Goff D. Glycemic index of foods: a physiological basis for carbohydrate exchange. Am J Clin Nutr. 1981;4: 362-6.

3. Petitot M, Boyer L, Minier C, Micard V. Fortification of Pasta with split pea and Faba Bean flours: Pasta processing and quality evaluation. Food Res Int. 2010; 43:634-41.

4. Suter, PM. Carbohydrates and dietary fiber. Handb Exp Pharmacol. 2005;170:231-61.

5. Murphy M, Douglass JS, Birkett A. Resistant Starch Intakes in the United States. J Diet Assoc. 2008;108:67-78.
6. Behall KM, Scholfield, DJ, Hallfrisch, JG, LiljebergElmståhl, HG. Consumption of both resistant starch and $\beta$-glucan improves postprandial plasma glucose and insulin in women. Diabetes Care. 2006 ; 29(5), 976-81.

7. Yamada $Y$, Hosoya S, Nishimura S, Tanaka T, Kajimoto $Y$, Nishimura A, Kajimoto O. Effect of bread containing resistant starch on postprandial blood glucose levels in humans. Biosci Biotechnol Biochem. 2005; 69(3), 559-66.

8. Noakes M, Clifton PM, Nestel PJ, Le Leu R, Mcintosh G. Effect of high-amylose starch and oat bran on metabolic variables and bowel function in subjects with hypertriglyceridemia. Am J Clin Nutr. 1996; 64(6), 944-51.

9. Lunn J, Buttriss JL. Carbohydrates and dietary fibre. Nutr Bull. 2007; 32(1), 21-64.

10. Ulmius $M$, Johansson $A$, Önning $G$. The influence of dietary fibre source and gender on the postprandial glucose and lipid response in healthy subjects. Euro J Nutr. 2009; 48(7), 395-402.

11. Granito M, Frías J, Doblado R, Guerra M, Champ M, VidalValverde $C$. Nutritional improvement of beans (Phaseolus vulgaris) by natural fermentation. Euro Food Res Tech. 2002; 214: 226-231.

12. Jenkins DJA, Ghafari $H$, Wolever TMS, Taylor RH, Jenkins AL, Barker HM, Fielden H, Bowling, AC. Relationship between rate of digestion of foods and post-prandial glycaemia. Diabetologia. 1982; 22(6), 450-5.

13. Tovar J, Björck IM, Asp NG. Incomplete digestion of legume starches in rats: A study of precookeed flours containing retrograded and physically inaccessible starch fractions. J Nutr. 1992; 122: 1500-7.

14. Tovar J, Granfeldt Y, Björck IM. Effect of processing on blood glucose and insulin responses to starch in legumes. J Agricul Food Chem. 1992; 40, 1846-51.

15. Cassab Carreira M, Lajolo M, Wenzel E. Glycemic Index: Effect of Food Storage under Low Temperature. Brazilian Arch Bio Tech. 2004; 47(4): 569-74.

16. Osorio-Díaz P, Agama-Acevedo E, Mendoza-Vinalay $M$, Tovar J, Bello-Pérez L. Pasta added with chickpea flour: chemical composition, in vitro starch digestibility and predicted glycemic index. Cienc Tecnol Aliment. 2008; 6(1): 6-12.

17. Liljeberg $H$, Elmsta $H$. Resistant starch content in a selection of starchy foods on the Swedish market. Euro J Clin Nutr. 2002;56: 500-60.

18. Landa-Habana L, Piña-Hernández A, Agama-Acevedo E, Tovar J, Bello-Pérez LA. Effect of Cooking Procedures and Storage on Starch Bioavailability in Common Beans (Phaseolus vulgaris L.). Plant Foods Hum Nutr. 2004;59(4):133-6.

19. Wolever TMS. Índice Glucémico. Clasificación fisiológica de los hidratos de carbono de la dieta. Editorial Acribia SA. España. 2006.

20. Jenkins DJA, Vuksan V, Kendall CW, Wush P, Jeffcoat $R$, Waring S, Mehling CC, Vidgen E., Augustin LS, Wong E. Physilogical effects of resistant starches on fecal bulk short chain fatty acids, blood lipids and glycemic index. J Am Col Nutr. 1998; 17:609-16.

21. International Pasta Organization (IPO). 2014. Pasta percapita consumption. Disponible en http://www.internationalpasta.org/index.aspx?idsub=118. Recuperado el 13 de enero de 2014.

22. Granito $M$, Ascanio $V$. Desarrollo y transferencia tecnológica de pastas funcionales extendidas con leguminosas. Arch Latinoam Nutr. 2009;59:71-7.

23. Abecassis J, Faure J, Feillet P. Improvement of cooking 
quality of maize pasta products by heat treatment. J Sci Food Agricul. 1989;47(4):475-85.

24. Matsuo RR, Bradley JW, Irvine GN. Effect of protein content on the cooking quality of spaghetti. Cereal Chem. 1972; 49:707-11.

25. Robinson HW, Hodgen CG. The biuret reaction in the determination of serum protein. I. A study of the condition necessary for the production of the stable which bears a quantitative relationship to the protein concentration. J Biol Chem 1940;135:707-11.

26. AOAC. Methods of Analysis [CD-ROM]. Washington (DC): Association of Official Analytical Chemists. 1 CD-ROM: sound, color, 4 3/4 in. 1990.

27. Goñi I, Díaz-Rubio M, Pérez-Jiménez J, Saura-Calixto F. Towards an updated methodology for measurement of dietary fiber, including associated polyphenols, in food and beverages. Food Res Inter. 2009;42:840-6.

28. Goñi I, García-Alonso A, Saura-Calixto F. A starch hydrolysis procedure to estimate glycemic index. Nutr Res. 1997;17:427-37.

29. Goñi I, García-Diaz L, Mañas E, Saura-Calixto F. Analysis of resistant starch: a method for foods and food products. Food Chem. 1996;56:445-9.

30. Wolever $T$, Jenkins $D$, Jenkins A, Josse $R$. The glycemic index: methodology and clinical implications. Am J Clin Nutr. 1991;54: 846-54.

31. Goñi l, Valentín-Gamazo C. Chickpea flour ingredient slows glycemic response to pasta in healthy volunteers. Food Chem. 2003;81:511-5.

32. Arteaga, A. El índice glicémico: una controversia actual. Nutr Hosp. 2006;21(2):55-60.

33. COVENIN. Comisión Venezolana de Normas Industriales. Pastas alimenticias (2da. revisión). Norma Venezolana
COVENIN 1994, 283. Caracas, Venezuela. p 12, 1994.

34. Zhao YH, Manthey FA, Chang SKC, Hou HJ, Yuan SH. Quality characteristics of spaghetti as affected by green and yellow pea, lentil, and chickpea flours. J Food Sci. 2005;70(6), S371-S376.

35. Martín MA, Gonzalez C, Alborz A. Cooking properties of fresh pasta supplemented with tiger nut flour. InsideFood Symposium. (Belgium), Communication No 82. 2013.

36. Chillo S, Laverse J, Falcone PM, Protopapa A, Del Nobile $M A$. Influence of the addition of buckwheat flour and durum wheat bran on spaghetti quality. J Cereal Sci. 2008;47(2):144-52.

37. Gueguen J, Barbot J. Quantitative and qualitative variability of pea (Pisum sativum L.) protein composition. J Sci Food Agric. 1988;42(3):209-24.

38. Tudorica CM, Kuri V, Brennan CS. Nutritional and Physicochemical Characteristics of Dietary Fiber Enriched Pasta. J Agric Food Chem. 2002;50:347-56.

39. Gallegos-Infante JA, Rocha-Guzman NE, Gonzalez-Laredo RF, Ochoa-Martínez LA, Corzo N, Bello-Perez LA, MedinaTorres L, Peralta-Alvarez LE. Quality of spaghetti pasta containing Mexican common bean flour (Phaseolus vulgaris L.). Food Chem. 2010;119:1544-9.

40. INN. Instituto Nacional de Nutrición. Tabla de composición de alimentos para uso práctico. Serie Cuadernos Azules. Caracas, Venezuela. Publicación No 52. 1999.

41. Arvidsson-Lenner R, Nils-Georg A, Axelsen M, Bryngelsson, S, Haapa E, Järvi A, Karlström B, Raben A, Sohlström $A$, Thorsdottir I, Vessby B. Glycaemic Index. Relevance for health, dietary recommendations and food labeling. Scandinavian J Nutr. 2004;48(2): 84-94.

42. Björck I, Liljeberg H, Östman, E. Low glycaemic-index foods. Brit J Nutr. 2000;83:S149-S55. 